\title{
Analysis of the multi-level battery management system functional index system weight
}

\author{
Mingsheng Chen ${ }^{1, \mathrm{a}}$, Zhimao Ming ${ }^{1, \mathrm{~b}}$, Guixiong Liu $^{2, \mathrm{c}^{*}}$, Yu Zhang ${ }^{2, \mathrm{~d}}$ \\ ${ }^{1}$ Guangzhou GRG metrology and test Co., Ltd, Tianhe, Guangzhou, Guangdong, China \\ ${ }^{2}$ School of Mechanical and Automotive Engineering, South China University of Technology, Tianhe, Guangzhou, Guangdong, China \\ aemail:chenms@grgtest.com, bemail:mingzm@grgtest.com,
}

\begin{abstract}
In order to identify index system property of battery management system, index system reconstruction and index weights reassignment are performed based on industrial and national standards, using multi-the analytic hierarchy process. The result shows that: compared to vehicle industry standard of seventeen technical indexes, new national standard have thirty-three indexes. And index system is rebuilt as two layers, where the first layer includes five indexes. By using of judge matrix, characteristic vector and consistent verify, all indexes weight and their consequences are confirmed. In the first layer, the index of performance of estimation, calculation and diagnose shows the maximum weight of 0.4283 , and electrical adaptability has the minimum weight of 0.0710 .
\end{abstract}

\section{Introduction}

With the increasing concern about the safety of battery management system in the electric vehicle industry, the industry standard[1] on the technical conditions of electric vehicle management system promulgated and implemented in 2011 has gradually failed to meet the pace of technology update[2], and the recommended national standard has been promulgated in 2020[3]. The current research mainly focuses on the development and application of the hardware function of Battery Management System (BMS) [4-6]. The hierarchical relationship between the functional and technical indexes of the BMS and the importance of each index are rarely studied. In this paper, multi-level analysis is used to study the hierarchical characteristics of the functional performance index system of the battery management system, the weight distribution of each index and its ranking, and to provide a technical basis for the safety evaluation of key components of electric vehicles[7].

\section{BMS function index system}

\subsection{Function type}

This section analyzes and reconstructs the standard index system hierarchy, taking into account the main functions of the battery management system, and lays the foundation for the subsequent research on weight calculation.

\subsection{System of evaluation indicators}

At present, the domestic automotive industry standard QC/T 897-2011 Technical Conditions of Battery Management System for Electric Vehicles has a comprehensive coverage of the functional indicators of the battery management system, but there are deficiencies in the testing methods of key indicators such as SOC estimation accuracy, voltage and temperature monitoring, such as the layout of no monitoring points and fuzzy hierarchy among items, etc. In this paper, a total of three indicators, such as state parameter measurement accuracy, SOC estimation accuracy and battery fault diagnosis performance, are divided into three levels and named. To address the above situation, this paper divides a total of three indicators such as state parameter measurement accuracy, SOC estimation accuracy and battery fault diagnosis, and names them as measurement accuracy and diagnostic performance. The remaining fourteen items are divided into hierarchical indexes such as Insulation performance, Electrical adaptability, Environmental performance and Electromagnetic compatibility, respectively.

For the recommended national standard GB/T 386612020 Technical Conditions of Battery Management System for Electric Vehicles, in order to achieve the hierarchical consistency of the whole index system, the seven items such as total voltage measurement accuracy, state parameter measurement accuracy, SOC estimation accuracy and battery fault diagnosis in the first level are grouped into one level again, and named as Measurement and Diagnosis. Performance. The reconstructed index has

*Guixiong Liu: 'email: megxliu@scut.edu.Cn, demail: 201821003079@mail.scut.edu.cn 
two levels of indicators and Table 1 shows the first level of indicators.

Table 1. Standard index level reconstruction

\begin{tabular}{|c|c|c|c|}
\hline \multirow{2}{*}{ Serial number } & \multirow{2}{*}{ First-level indicators } & \multicolumn{2}{|c|}{ Number of second-level indicators } \\
\hline & & QC/T 897 & GB/T 38661 \\
\hline 1 & $\begin{array}{l}\text { Measurement accuracy and } \\
\text { diagnostic performance }\end{array}$ & 3 & 7 \\
\hline 2 & Insulation performance & 2 & 2 \\
\hline 3 & Electrical adaptability & 2 & 7 \\
\hline 4 & Environmental performance & 8 & 10 \\
\hline 5 & $\begin{array}{l}\text { Electromagnetic } \\
\text { compatibility }\end{array}$ & 2 & 7 \\
\hline
\end{tabular}

\section{Multilevel weighting analysis methods}

\subsection{Weighting analysis method}

Among the commonly used weight analysis methods, the subjective assignment method has the advantage of determining the weight according to the meaning of the attribute itself, but is less objective; the objective assignment method has the advantage of determining the weight without considering the actual meaning of the attribute, but sometimes the determined weight may contradict the actual importance of the attribute. The algorithm and requirements of the comprehensive analysis method are relatively high and not very practical. In view of the multi-layered nature of the functional safety index evaluation system of the battery management system, this paper adopts the analytic hierarchy process to assign weights. Analytic hierarchy process takes a complex multi-objective decision-making problem as a system, decomposes the objective into multiple objectives or guidelines, and then into several levels of multiple indicators (or guidelines, constraints), and then calculates the hierarchical single ranking (weights) and total ranking through qualitative indicator fuzzy quantification methods, in order to serve as a systematic method for objective (multi-indicator) and multi-scheme optimization decisionmaking. The advantages are: systematic analysis method, simple and practical decision-making method, and less quantitative data information required.

\section{2 multilevel computational model}

Multilevel weighting analysis process, build a hierarchical model,construct a pairwise comparison array,compute the weight vector and consistency test,compute the combined weight vector and consistency test. The establishment of the progressive hierarchy model has been established in the aforementioned system of indicators and will not be repeated here. Criteria for constructing the judgment matrix of each level: use the scalar method to quantify the influence of two indicators[8]. By column vector normalization, after summing and solving the weight vector and maximum feature vector by row, you can calculate the synthetic weights of the indicators at each level on the system's goals and rank them overall to determine the importance of each indicator at the bottom of the hierarchical structure in the overall goal. The consistency indicators and their ratios are calculated, and it is usually considered that the consistency of the judgment matrix is acceptable when the consistency ratio is less than 0.1 , otherwise the judgment matrix should be appropriately modified. The specific calculation model is shown below[9-10].

Column vector normalization:

$$
\tilde{w}_{i j}=a_{i j} / \sum_{i=1}^{n} a_{i j}
$$

Where $w_{i j}$ is Vector normalized to row $i$ and column $j$, $a_{i j}$ is indicator values for row $i$ and column $j, n$ is matrix degree.

Row sum:

$$
\tilde{w}_{i}=\sum_{j=1}^{n} \tilde{w}_{i j}
$$

Where $w_{i}$ is Vector value after summation in row $i$.

Calculating the maximum eigenroot approximation:

$$
\lambda=\frac{1}{n} \sum_{i=1}^{n} \frac{(A w)_{i}}{w_{i}}
$$

Where $A$ is judgment matrix, $W$ is eigenvector, $\lambda$ is maximum eigenvalue root approximation.

Consistency testing:

$$
C I=\frac{\lambda_{\max }(A)-n}{n-1}
$$

Where $C I$ is Judgment Matrix Consistency Indicator, $\lambda_{\max }$ is The mean of the largest characteristic roots. 


\section{Weight of the functional indicator system}

\subsection{Judgment matrix and consistency}

Table 2 shows the results of constructing a judgment matrix combining applications and statistics. For ease of representation, the hierarchy is abbreviated as $\mathrm{x}, \mathrm{x}=\mathrm{A}, \mathrm{B}$, C, D, E

Table 2. First-level indicator judgement matrix

\begin{tabular}{cccccc}
\hline serial number & A & B & C & D & E \\
\hline A & 1 & 5 & 2 & 3 & 4 \\
B & $1 / 5$ & 1 & $1 / 3$ & $1 / 2$ & $1 / 2$ \\
C & $1 / 2$ & 3 & 1 & $1 / 2$ & $1 / 3$ \\
D & $1 / 3$ & 2 & 2 & 1 & 2 \\
E & $1 / 4$ & 2 & 3 & $1 / 2$ & 1 \\
\hline
\end{tabular}

Table 3 shows the hierarchical consistency results obtained using equations (1) (4).

Table 3. Eigenvector and consistency ratio

\begin{tabular}{ccc}
\hline parameters & first level & note \\
\hline eigenvector & 5.4220 & $\lambda$ \\
coherence ratio & 0.0942 & $C I$ \\
\hline
\end{tabular}

According to the consistency determination criteria, the consistency ratio should be less than 0.1 . From the calculated statistical results in Table 3 , it can be seen that all levels of consistency meet the requirements.

\subsection{Weights and ranking}

Table 4 shows the results of the weighting and ranking of the indicators.

Table 4 Indicator weighting and ranking

\begin{tabular}{cccc}
\hline Serial number & First-level indicators & weights & sequence \\
\hline 1 & Measurement accuracy and & 0.4283 & 1 \\
2 & diagnostic performance & 0.0710 & 5 \\
3 & Insulation performance & 0.1319 & 4 \\
4 & Electrical adaptability & 0.2003 & 2 \\
5 & Environmental performance & & 3 \\
\hline
\end{tabular}

\section{Conclusions}

In this paper, a quantitative study of the weights of the functional performance technical indicators of the battery management system was carried out by constructing a multilevel judgment matrix, weighting calculations and consistency testing methods. The main conclusions are:

(1) On the basis of the automotive industry standard, the newly promulgated national recommended standards have re-divided the hierarchy of functional technical indexes of battery management system into thirty-three technical indexes. In this paper, a total of seven items such as state parameter measurement accuracy, SOC estimation accuracy and battery fault diagnosis are integrated into the measurement and diagnosis performance category to realize the new index system hierarchy.

(2) The construction of the judgment matrix is particularly critical and directly affects the weight of the indicators. Although the initial conditions established in this paper have some generality, they may not meet the needs of extreme environments and special situations such as vacuum and high pressure.

(3) Multi-the analytic hierarchy process is used to obtain the weight distribution of indicators at each level and the consistency test at each level, and the importance of different functional performance indicators in the research and application work can be clarified according to the ranking of weights.

\section{Acknowledgments}

This paper is one of the stage results of the Guangdong Key Areas R\&D Project Power Battery System Testing and Evaluation Technology Research (2019B090908003). 


\section{References}

1. Technical conditions of battery management system for electric vehicles: QC/T 897-2011.

2. Pu, F.T. (2016) Nine functions of pure electric vehicle battery management system. Automotive electrical appliances, 1:67-68.

3. Technical conditions of battery management system for electric vehicles: GB/T 38661-2020.

4. Xiang, J.L. (2012) Research on Large Capacity Power Lithium Battery Management System (BMS). Hefei University of Technology, Hefei.

5. Tan, X.J. (2011) Design of Electric Vehicle Power Battery Management System. Zhongshan University Press, Guangzhou.

6. Andrea, D. (2010) Battery Management Systems for Large Lithium-Ion Battery Packs. Artech House Publishers, London.

7. Dong, S.G. (2003) Realization of Analytic Hierarchy Process Based on MATLAB. Aviation weapons, 4:16-18.

8. Guo, J.Y, Zhang Z.B, Sun Q.Y. (2008) Research and Application of AHP. China Safety Science Journal,18: 148-153.

9. Cui, J.X, Li Q.X.(2014) Research on and application of improved multilevel analysis. Mathematical practice and understanding,44: :217-222.

10. Li, Z.Y.(2012) Research on the Application of Hierarchical Analysis in Multilevel Multiple Indicator Assessment Systems (MMIAS). Journal of Huaihai University of Technology,02:73-76. 